\title{
Adaptive Control of Satellite System using Kalman Estimates in the presence of Disturbance
}

\author{
D. Y. Dube, H. G. Patel \\ Sardar Vallabhbhai National Institute of Technology \\ Surat-395007, India \\ deepalidube404@gmail.com; hpatel.svnit@gmail.com
}

\begin{abstract}
There has always been an increasing interest in the space related activities. The research on aerospace vehicles have become popular due to the problems and complexity of the systems involved. The main challenge lies in keeping a balance when in motion and hence tracking plays a key role here. The satellite system discussed in this paper is described for the problem arising due to presence of disturbances (colored and white noise). This model is evinced by the augmentation of discrete Kalman filter, a powerful tool for controlling the noisy estimates. Whereas, the regulation of the state dynamics (Euler angles and velocity) is achieved with the adaptive law which focuses on guaranteeing stability of adaptive control scheme using a good choice of Lyapunov argument. The presence of Gaussian White noise is also mentioned where a comparison resembles stability for almost, mean square, third moment and mean value between the satellite and oscillator system. A clear discussion of the system in the presence of colored and white noise with perfect estimates derived from kalman filter and an adaptation law governing the evolution of system around equilibrium is done.
\end{abstract}

Keywords: Adaptive control, Colored noise, Discrete Kalman filter, Gaussian white noise.

\section{Introduction}

The expertise required for governing the inclination of a satellite and its dominance for pointing in a desired order is a part of spacecraft attitude determination and control. Also, the demand for almost all space assignments are convinced by proposal of attitude determination and control systems distinctly. Attitude control is essential in almost all space missions. Their objectives may be disrupted without attitude control. The Kalman filter has been applied for attitude control to serve the problem of fault detection, diagnosis and isolation (FDDI) in interaction with multiple model filters for both partial and total reaction wheels [1]. For road tracking, the images received from satellite are the initial source of information. A combination of EKF and PF algorithm was adopted for better navigation [2].

There are systems to be used for world-wide real-time positioning, data collection and requires an active target satellite. Such demands were fulfilled by designing of strong algorithm. Hence, a multi-model kalman filter and a redundant adaptive robust EKF discussed by [3,4] proved to be a unique solution. The requirements of attitude determination, finite-time attitude tracking in space missions for the conditions where exogenous disturbances and inertia uncertainties degrade the performance was addressed. Such issues were visited by $[5,6]$ with the strategy of extended kalman with gain scheduled in nano-satellite for determining attitude and a novel adaptive sliding mode control for robustness, faster response and high precision control was implemented.

A similar filter was used by $[7,9,11]$ to provide autonomous orbit determination capability for the satellite refraction navigation system. the unscented transformation principle was utilized to transform the measurement noise and its covariance matrix. Whereas, a new range of uncertainties, parameter variations or noises with sufficient conditions and a lagrange multiplier made the idea successful for formation flying of satellite. [8] made the use of a novel predictive smooth variable structure filter (PSVSF) to accommodate the enhanced tracking.

Until now several governing laws were used because of their benefits in tracking. An adaptive sliding mode control leading to an asymptotic stability and autonomous flight of satellite was considered by [10]. A simplified nonlinear model having the dynamics in Euler-Lagrange form was investigated by [12] where the fractional order operator and saturation function into the sliding surface lead to the evolution of a new adaptive function. 
The dynamic stability of a two-dimensional system under bounded noise excitation was studied by [14]. This bounded noise later became white noise and its characteristic was evolved through the determination of the $\$ \mathrm{p}^{\wedge}\{$ th $\} \$$ moment and the principle Lyapunov exponent. The similar problem of presence of white noise in the dynamic system was dealt by a numerical method [15]. It was concluded that the "periodic windows" or intervals of the largest Lyapunov exponent beyond the onset of chaotic motion are gradually diminished when the intensity of bounded noise is increased. Another approach on the two degree-of-freedom linear systems [16] was presented and better response of stability from Lyapunov and moment Lyapunov were recorded. Therefore, so far the disturbance due to colored noise has not been focused. The novelty of this study lies in revealing the behavior of the satellite dynamics in the presence of both colored and white noise when in motion and improving the governing action with application of advance control scheme.

In this paper, the problems occurring due to the presence of noisy disturbance (colored) are simplified in Section II. A mathematical representation of the system dynamics in Section III provides an easy understanding for the concerned variables. Design of Kalman filter and adaptive control law is discussed in Section IV and V. A gaussian white noise excitation on the system dynamics provides a sound relation of various stability criterions in Section VI. Simulation results in section VII makes the proposed technique more appealing with the final conclusion quoted in Section VIII.

\section{Problem Formulation}

There is observation regarding brisk movements on earth's airspace density with intrusion. Such swings are included in deterministic models. One such model arose from the problem of stabilizing a satellite in a circular orbit and is studied by Balakrishnan [17]:

$$
I \dot{x}+x \otimes I x+K x=D \xi_{t}
$$

where $x$ is the 3-dimensioonal state vector, $I, K$ and $D$ is the real symmetric positive definite matrices, $\xi_{t}$ is a 3 dimensional colored noise. Also, $I$ is the moment of inertia matrix, $K$ is a friction matrix and $D$ the noise intensity and correlation matrix. The above equation is modeled as 3-dimensional vector Ito stochastic differential equation

$$
d X_{t}=f\left(X_{t}\right) d t+I^{-1} D d W_{t}
$$

The problem due to the atmospheric distractions or swings were modeled in (3-5). Above equations were evolved virtually in MATLAB with a 3-dimensional Ito-stochastic differential equation. The weiner process $\$ \mathrm{~W}(\mathrm{x}) \$ \mathrm{being}$ analysed with randomness in the vector (Brownian motion). This concluded a different output each time the system is manipulated. Atmospheric chaos are necessary for design of both inlet and engine flight controls and for studying link between the propulsion and the vehicle structural dynamics for supersonic vehicles. In the referred paper, a more accurate model was developed by George to represent fractional order of atmospheric disturbances. Atmospheric model was accomplished by first ascending the kolmogorov spectral to convert them into finite energy von-karman forms. Hence, the objective lies for given parameters and the atmospheric conditions with all prior information, (the poles and zeros describing disturbances for respective acoustic velocity, temperature, pressure and density) the appropriate time domain simulations are evaluated. These disturbances also contribute to the problems cited in this paper. Their respective transfer functions as in [18]:

$$
\begin{aligned}
& G_{L A}(s)=\frac{(s / 9.2+1)(s / 55+1)(s / 335.5+1)}{(s / 1.46+1)(s / 30.1+1)(s / 85.7+1)(s / 1593.1+1)} \\
& G_{T}(s)=\frac{(s / 33+1)(s / 45.6+1)(s / 602.4+1)}{(s / 1.1+1)(s / 25.1+1)(s / 109.8+1)(s / 816.3+1)} \\
& G_{P}(s)=\frac{(s / 33+1)(s / 45.6+1)(s / 602.4+1)}{(s / 1.1+1)(s / 25.1+1)(s / 109.8+1)(s / 816.3+1)}
\end{aligned}
$$


here $G_{L A}, G_{T}$ and $G_{P}$ are the atmospheric disturbance transfer function for longitudinal, temperature and pressure. These exhibit behavioral pattern when in the system and may cause large instability in satellite control system operations. As shown in Fig. 3, the response is completely unwanted. It reflects that the disturbance is very inclined in the right half plane. A specific scaling will allow the satellite to hover at an altitude for executing spinning maneuver and also communicate the required messages through sensing.

\section{Satellite System}

For the perceptible scrutinization of the attitude dynamics and control of spacecraft, a vague satellite is considered. This is a three-axis preserved system which is visualized as either a MIMO coupled dynamical system or as a SISO decoupled dynamical system provided each axis is self-reliant of others having no communications among them. The three control torques $\tau_{1}, \tau_{2}, \tau_{3}$ in each of the three axes. Fig. 1 is the schematic diagram of the system under consideration. The attitude dynamics given for the satellite motion is of the following first-order non-linear differential equations:

$$
I \dot{x}+x \otimes I x+K x=D \xi_{t}
$$

where for instance we take

$$
I=\left[\begin{array}{ccc}
22 & 0 & 0 \\
0 & 15 & 0 \\
0 & 0 & 22
\end{array}\right],
$$

here $x=\omega=\left[\begin{array}{lll}\omega_{x} & \omega_{y} & \omega_{z}\end{array}\right]^{T}, K=\left[\begin{array}{lll}K_{u x} & K_{u y} & K_{u z}\end{array}\right]^{T}$ which resembles $I, x=\omega, K_{u}$ and $\xi_{t}$ as the satellite's inertial matrix, the state vector, friction matrix and $D \xi_{t}$ is the colored noise. The inertia tensor about the center of gravity as given above comprises of

$$
I=\left[\begin{array}{ccc}
I_{x} & -I_{x y} & -I_{x z} \\
-I_{x y} & I_{y} & -I_{y z} \\
-I_{x z} & -I_{y z} & I_{z}
\end{array}\right]
$$

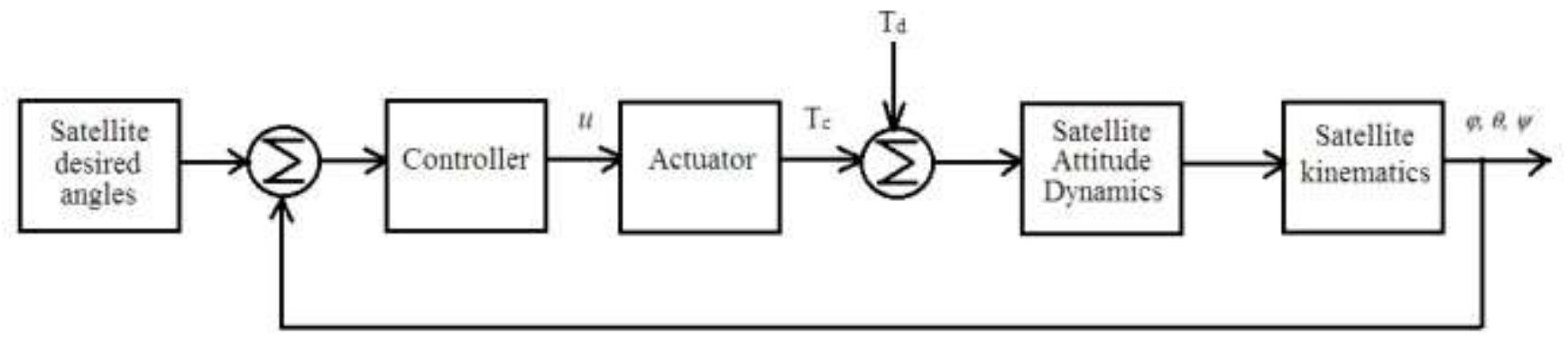

Fig. 1: Satellite simulation diagram. $T_{d}$ is the disturbance and $T_{c}$ is the control torque.

In case, if the Euler angles are used to resemble attitude, the kinematics will be:

$$
\dot{\theta}=J(\theta) x
$$


where $\theta=\left[\begin{array}{lll}\Phi & \Theta & \Psi\end{array}\right]^{T}$ and

$$
J(\theta)=\left[\begin{array}{ccc}
1 & \sin \Phi \tan \Theta & \cos \Phi \tan \Theta \\
0 & \cos \Phi & -\sin \Phi \\
0 & \sin \Phi / \cos \Theta & \cos \Phi / \cos \Theta
\end{array}\right]
$$

Considering $\cos \Theta=0$. In Eq. (6), if the control moments are considered then it will be simplified as

$$
I \dot{x}+x \otimes I x+K x=D \xi_{t}+\tau
$$

here $\tau=\left[\begin{array}{lll}\tau_{1} & \tau_{2} & \tau_{3}\end{array}\right]^{T}$ is evolved by implementing actuators. It is possible to linearize the kinematic relationship in terms of the euler angles $(\phi, \theta, \psi)$ of the satellite rigid body model (body fixed frame). Substitution of linear kinematic theory conducts the relationship as below in Eqs. (12-14). Also, Fig. 2 gives the plan about the satellite dynamics to be formed in simulink (MATLAB) for computation.

$$
\begin{gathered}
\ddot{\psi}+\frac{p_{1}}{I_{x}} \dot{\psi}+\left(1+\frac{\dot{h}}{I_{x}} \xi\right) \sin x+\frac{1}{2} \frac{h \dot{\alpha}}{I_{x}} \sin 2 x-\frac{T_{x}}{I_{x}}=0 \\
\ddot{\phi}+\frac{p_{3}}{I_{y}} \dot{\phi}+\left(1+\frac{h \omega_{z} \xi}{I_{y}}\right) \sin y-\frac{T_{y}}{I_{y}}=0 \\
\ddot{\theta}+\frac{I_{x z}}{I_{z}} \omega_{0}^{2} \dot{\theta}-\frac{h}{I_{z}} \xi \sin z \omega_{y}-\frac{T_{z}}{I_{z}}=0
\end{gathered}
$$

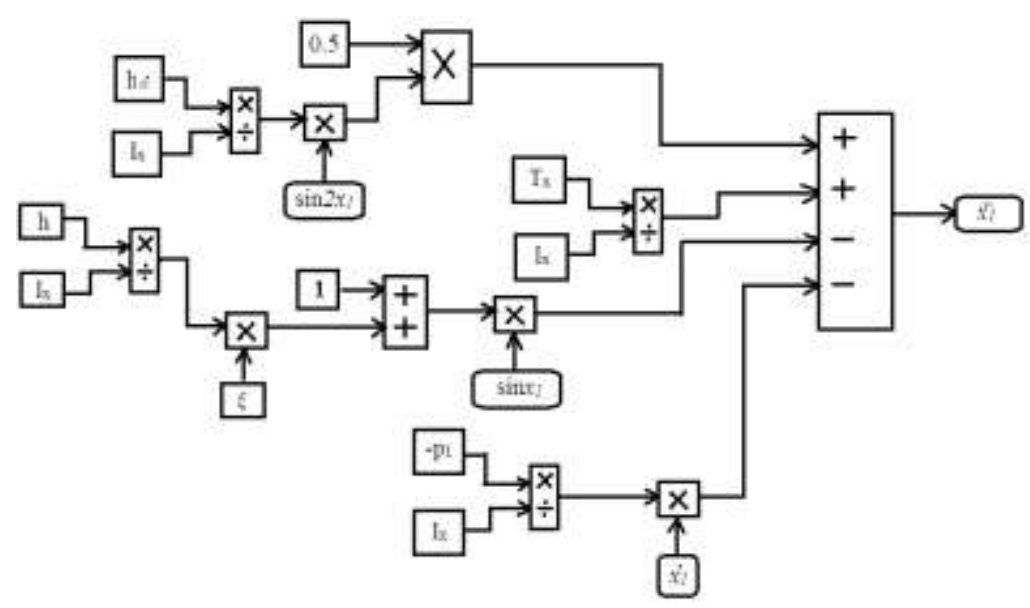

Fig. 2: Evolution of System dynamics in simulink as mentioned in Eq. (12).

\section{Kalman Filtering}

R. E. Kalman elaborated this filter in 1960. Due to advances in the evolution of digital computing, the kalman filter is a subject of extensive research and application. Kalman filtering has been put in application in the areas of aerospace, navigation, manufacturing and many others. The Kalman filter can be used for inference of missing information from 
indirect (and noisy) measurements. In designing filter for spacecraft navigation, the state $\left(X_{k}\right)$ can be obtained. Also, if discrete measurements are taken whether they come from a discrete or continuous system, the discrete kalman filter can be used. Because of today's advanced microprocessor technology and the fact that microprocessors can provide greater accuracy and computing power, hence digital control is being used more frequently instead of the classical analog control methods. This means that for modern control applications, the discrete Kalman filter is usually used. The kalman filter design involves for the discrete version of the plant

$$
x_{k}=A x_{k-1}^{+}+B u_{k}+\xi_{k}
$$

The iterative equations required to process the available data set each time needs to

- To cipher the Kalman gain,

- To cipher new estimate,

- To find new error in estimate.

However, the Kalman gain is a combination of

$$
L=\frac{E_{\text {est }}}{E_{\text {est }}+E_{\text {meas }}}
$$

A larger value of gain means that the error in the estimate is large than the error in the measured value. The new or current estimate is obtained by

$$
E_{e s t_{k}}=E_{e s t_{k-1}}+L\left(\text { Meas }-E_{e s t_{k-1}}\right)
$$

Finally the error in the estimate is calculated using

$$
E_{e s t_{k}}=(1-L) E_{e s t_{k-1}}
$$

The main objective of this filter to slowly zero in the error and reach quickly at the estimated or correct value. Therefore, for the said system the updated estimate being

$$
\hat{x}_{k}=\hat{x}_{k-1}+L\left(y_{k}-C \hat{x}_{k-1}\right)
$$

with the error in the estimate

$$
\hat{x}_{k}=(1-L) \hat{x}_{k-1}
$$

\section{Adaptive Law}

In adaptive control, a natural candidate is the plant placed in closed loop with a stabilizing known-parameter. For evaluating the stability of the satellite dynamics the following Lyapunov function candidate is suggested

$$
V=\frac{1}{2} x^{T} I x+g(\theta)
$$

where $g(\theta)$ is a positive definite function relying on the attitude. By differentiating $V$ with respect to the time gives: 


$$
\begin{aligned}
\dot{V} & =x^{T} I \dot{x}+\dot{\theta}^{T} \frac{\delta g}{\delta \theta} \\
& =x^{T}(-S(x) x+\tau)+x^{T} J^{T}(\theta) \frac{\delta g}{\delta \theta}
\end{aligned}
$$

Since $S(x)=-S^{T}(x)$, it follows that

$$
x^{T} S(x) x=0, \forall x
$$

This suggests that the control input $u$ should be chosen as

$$
\dot{V}=\left(x^{T}\left(u+J^{T}(\theta) \frac{\delta g}{\delta \theta}\right) \leq 0\right.
$$

The control law satisfying this is

$$
u=-R_{d} x-J^{T}(\theta) \frac{\delta g}{\delta \theta}
$$

where $R_{d}>0$. This finally gives

$$
\dot{V}=-x^{T} R_{d} x \leq 0
$$

and thus the stability and convergence follow from standard Lyapunov techniques.

\section{Gaussian white noise excitation}

An autocorrelation function $R_{W W}(\tau)=2 \pi \sigma^{2} \delta(\tau)$ for the white noise process $W(t)$, where $\tau$ is the time lag is introduced. The PSD $S_{W W}(\omega)=\sigma^{2}$ for an infinite variance [13]. Here the damping coefficient $\zeta_{0}$ is in terms of $\frac{p_{1}}{I_{x}}$. This is so because the damping of satellite oscillations can be achieved by changes of moment of inertia. With respect to the almost sure stability, the top Lyapunov exponent for Eq. (12) written as

$$
\ddot{x}(t)+\frac{p_{1}}{I_{x}} \dot{x}(t)+\left(1-\frac{T_{x}}{I_{x}} V(t)\right) x(t)=0
$$

is computed exactly:

$$
\lambda_{x}=\frac{p_{x}}{I_{x}}-\frac{\pi \sigma^{2}}{\left(1+\sqrt{1-\left(-\frac{p_{1}}{I_{x}}\right)^{2}}\right)^{2}}
$$


Equating Eq. (29) to zero, the stability criterion is obtained as

$$
\sigma_{c r}^{2}=-\frac{p_{x}}{I_{x}}\left(2+2 \sqrt{1-\left(-\frac{p_{1}}{I_{x}}\right)^{2}}-\left(-\frac{p_{1}}{I_{x}}\right)^{2}\right)
$$

Using a steady state value of power spectral density (PSD) across the frequency axis, the moment stability criteria are befitted. The four variations in the graph depict the stability in the sense of critical damping of the satellite system. The linear curve in $\zeta_{0}$ provides highest peak of almost sure stability. It is observed from the graph that the stability region deflates as the order of the moment increases. Also, the almost sure stability criterion foresees a stability region vast than those of the moment stability criterion (Fig. 9). The ordinates of the antecedent are almost double in favour to the ordinates of the square stability criterion.
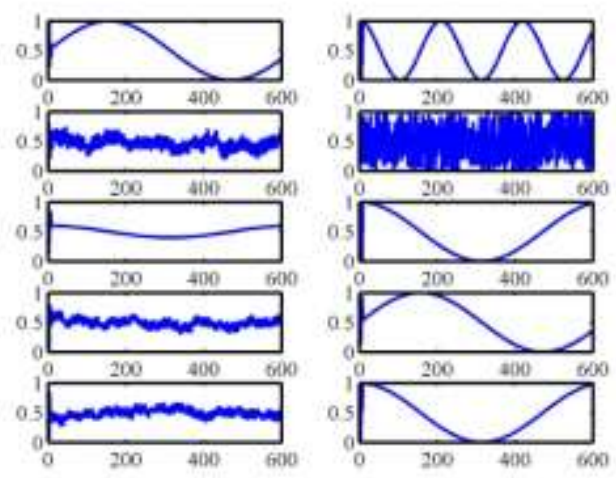

Fig. 3: The affect of stochastic disturbance at the output (Right hand side) via input (left hand side) of the satellite system.
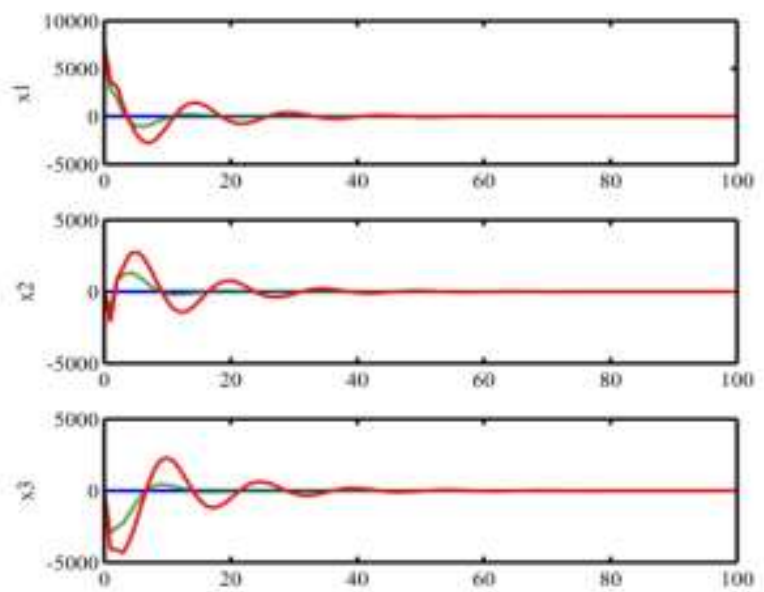

Fig. 4: Estimation using Kalman filter for satellite dynamics showing the correct estimate (Green), the corrected estimate (Blue), and for contrast, the uncorrected open loop model trajectory (Red). 

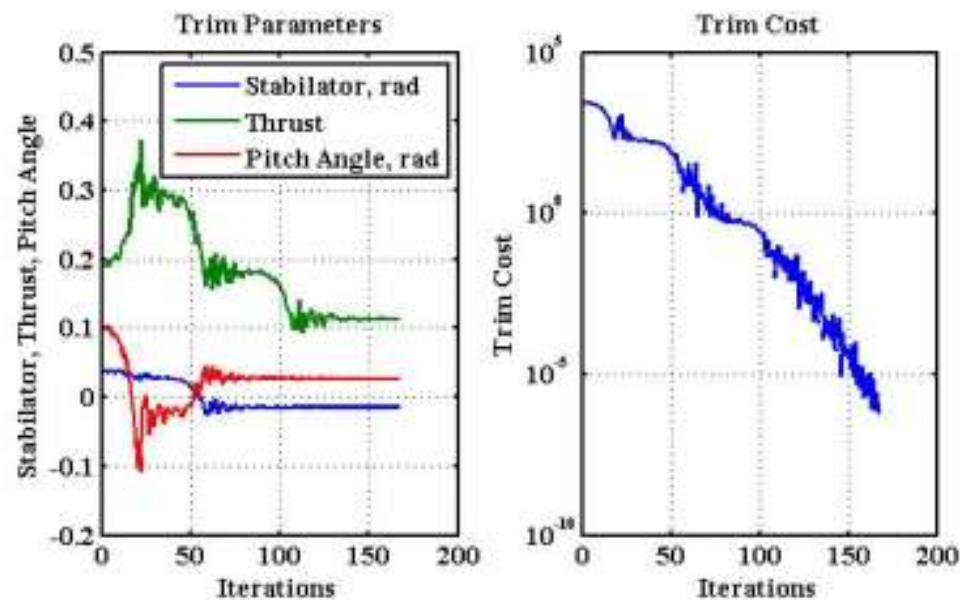

Fig. 5: Thrust, Stabilator and Pitch angle plot after filtering and governing.
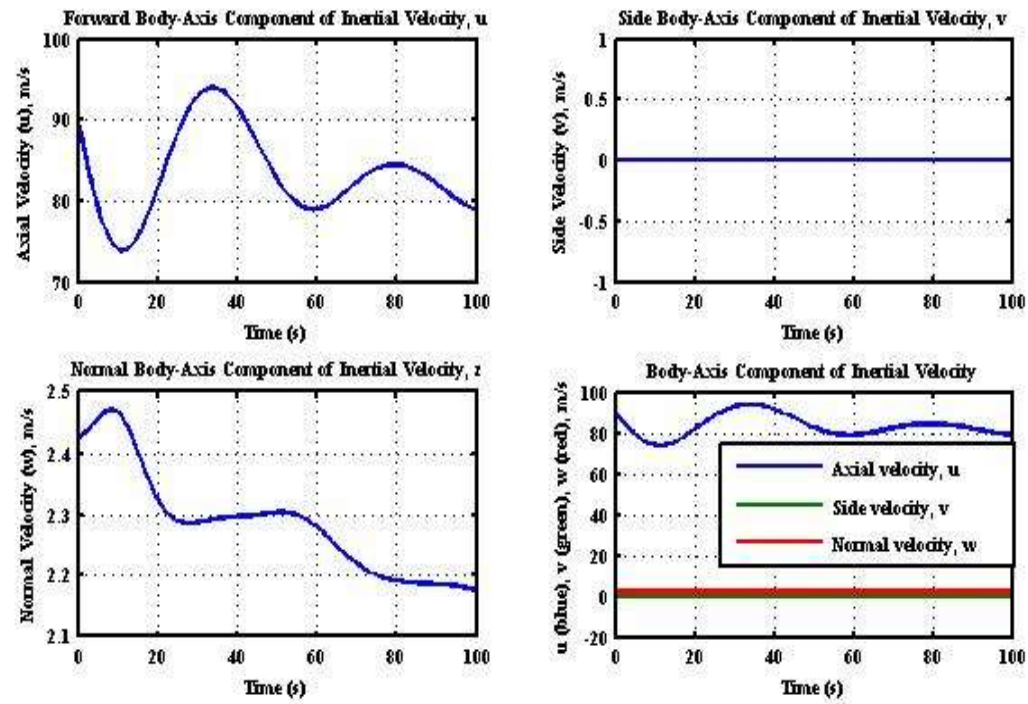

Fig. 6: Inertial velocity plot of satellite system in body fixed frame.
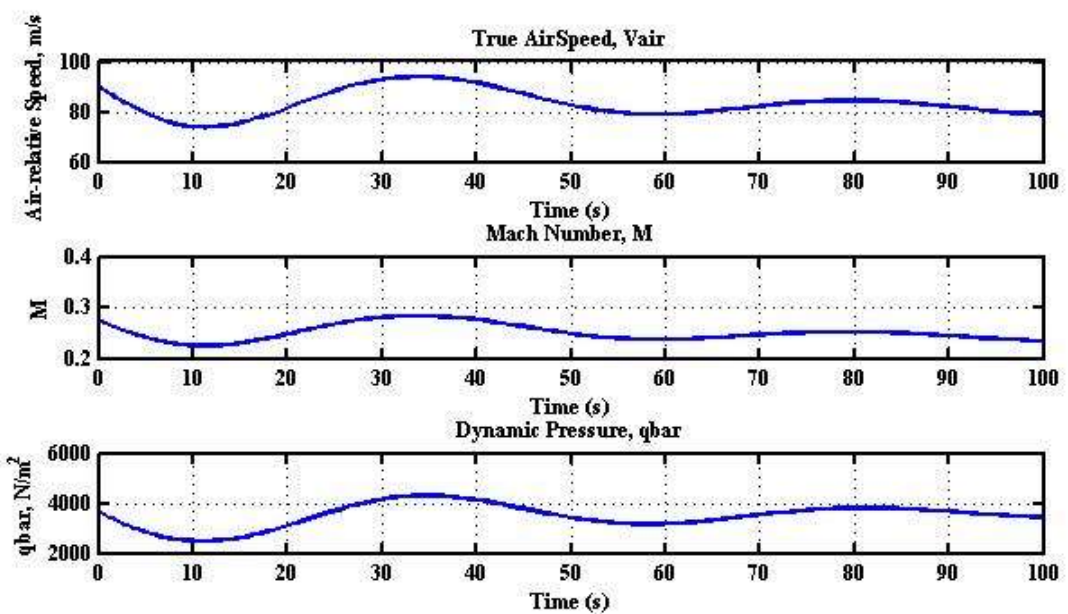

Fig. 7: The true airspeed, mach number and dynamic pressure curve of the spacecraft system. 

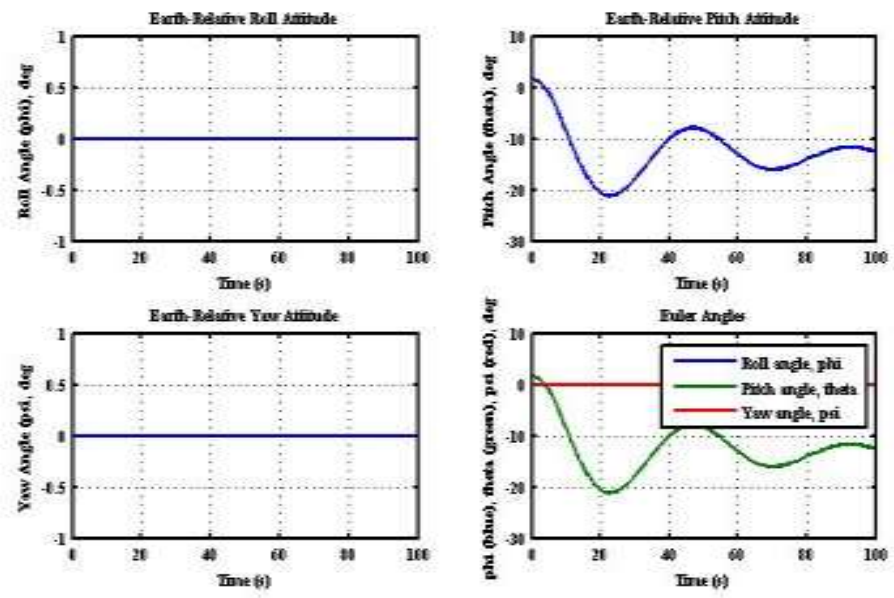

Fig. 8: Euler angle plot in Earth relative frame for satellite system.

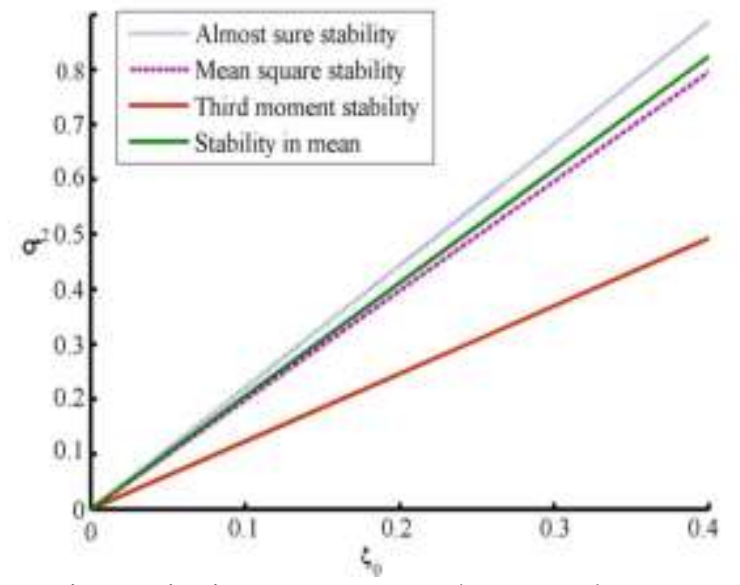

Fig. 9: The stability criteria for white noise excitation. From top to bottom: almost sure stability, stability in mean, mean square and third moment stability for satellite system.

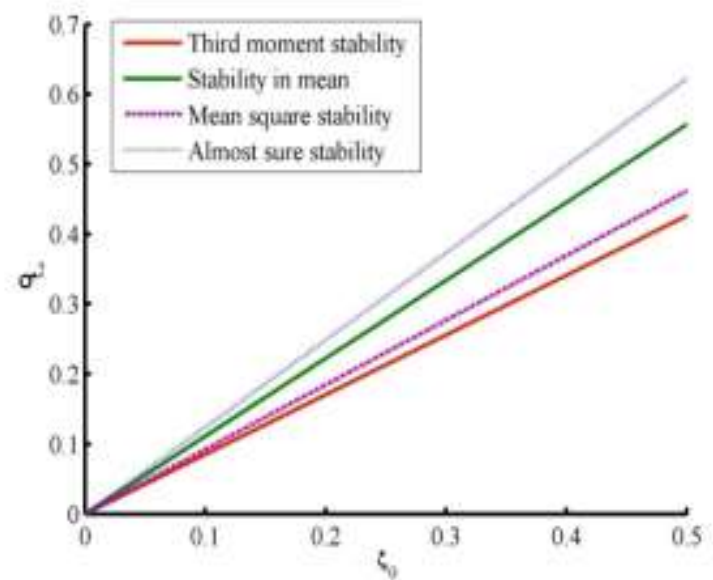

Fig. 10: The stability criteria for white noise excitation. From top to bottom: almost sure stability, stability in mean, mean square and third moment stability for oscillator system [13].

\section{Simulation Results}


The simulations are carried out to position the satellite at $90^{\circ}$ (one quarter revolution). The input provided to the system is in degrees with a response explaining the revolutions in radians for a zero at 0.8 and a pole at 0.2 as the lead compensator into the system. From Fig. 3, it is observed that the presence of atmospheric disturbances create an abnormal curve on the behavior of input-output estimates. Hence, there lies an urgent need of suppression of such stochastic noisy measurements from the system. The kalman estimates represent tracking of the velocities in $x, y$ and $z$ axes which is also described by states $x_{1}, x_{2}$ and $x_{3}$ in Fig. 4. It produces estimate of system's next state from the given estimate of systems current state, noisy sensor data, the possible model of systems dynamics and control commands. The stabilator, pitch angle and thrust variable is made noisy and in steady mode for successive iterations (Fig. 5). Fig. 6 and Fig. 8 resemble an inertial velocity curve in body fixed frame and an euler angle plot in earth relative frame of satellite system. In Fig. 6 the zaxis is parallel to the earth rotation axis and points towards the North pole. Whereas, the $\mathrm{x}$-axis is parallel to the line connecting the center of the earth with vernal equinox and points towards vernal equinox with zero side body axis component. The parameters like true air speed, mach number and dynamic pressure is recorded in Fig. 7 whereas, Fig. 9 and Fig. 10 provide a comparison of stability criteria for white noise excitation for an oscillator and satellite system. The stability of satellite attitude dynamics was scrutinized by availing Lyapunov's method. The boundary conditions being the dynamics returning to their native values with succeeding disturbances. The discrete kalman filter have proved to be a good choice, as it allowed the error to immediately reach to zero and gave exact value of true estimates. The adaptive control gave immense benefit for maintaining the angular velocity in both body and earth fixed frame. Whereas, the comparison of the white noise excitation given to the satellite and oscillator system gave an overview of the type of response achieved due to the perfect choice of Lyapunov exponent and design of stability criterion.

\section{Conclusion}

In this paper, the satellite system has been described and applied for the synthesis. The discussed controller and the collation with the filtering approach have been emphasized. It's properties are applied to position the satellite considering angle as an input. The second order continuous system known to be time-varying have been cast-off in this paper. Also, simulation results predict better response for the said problem for atmospheric disturbances of stochastic nature. The depicted controller indicates the competent capability for the said problem. The disturbances considered are of two main classes - colored and white noise. Their presence have off-course given a poor performance and an unstable orientation in space. Hence, to avoid problems due to bad orientation is addressed by the design of adaptive law in combination to filtering which helped to zero in the error estimates and lead to a satisfied convergence of the body parameters. The main advantage is the esteemed stability achieved for the tracking of pitch angle, thrust, earth-relative euler angles and velocity response.

\section{References}

[1] N. Tudoroiu and K. Khorasani, "Satellite Fault Diagnosis using a Bank of Interacting Kalman Filters", IEEE Trans. on Aerospace and Electronic Systems, Vol. 43, No. 4, October 2007, pp. 1334-1350.

[2] S. Movaghati, A. Moghaddamjoo and A. Tavakoli, "Road Extraction from Satellite Images Using Particle Filtering and Extended Kalman Filtering", IEEE Trans. on Geoscience and Remote Sensing, Vol. 48, No. 7, July 2010, pp. 28072817.

[3] R. Lopez, J. P. Malarde, F. Royer and P. Gaspar, “Improving Argos Doppler Location Using Multiple-Model Kalman Filtering", IEEE Trans. on Geoscience and Remote Sensing, Vol. 52, No. 8, August 2014, pp. 4744-4755.

[4] Y. Ali, Z. Jing and G. Liu, "'Maneuver-Aided active Satellite Tracking using Six-DOF Optimal Dynamic Inversion Control ", IEEE Trans. on Aerospace and Electronic Systems, Vol. 50, No. 1, Jan 2014, pp. 704-719.

[5] M. D. Pham, K. S. Low, S. T. Goh and S. Chen, "Gain-Scheduled Extended Kalman Filter for Nanosatellite Attitude Determination System", IEEE Trans. on Aerospace and Electronic Systems, Vol. 51, No. 2, April 2015, pp. 1017-1028.

[6] L. Chen, Y. Yan, C. Mu and C. Sun, "Characteristic Model-based Discrete-time Sliding Mode Control for Spacecraft with Variable Tilt of Flexible Structures", IEEE/CAA Journal of Automatica Sinica, Vol. 3, No. 1, Jan 2016, pp. $42-50$.

[7] X. Sun, P. Chen, C. Macabiau and C. Han, "Autonomous Orbit Determination via Kalman Filtering of Gravity Gradients", IEEE Trans. on Aerospace and Electronic Systems, Vol. 52, No. 5, Oct 2016, pp. 2436-2451. 
[8] L. Cao, Y. Chen, Z. Zhang, H. Li and A. Misra, "Predictive Smooth Variable Structure Filter for Attitude Synchronization Estimation During Satellite Formation Flying", IEEE Trans. on Aerospace and Electronic Systems, Vol. 53, No. 3, June 2017, pp. 1375-1383.

[9] X. Ning, F. Wang and J. Fang, “An Implicit UKF for Satellite Stellar Refraction Navigation System", IEEE Trans. on Aerospace and Electronic Systems, Vol. 53, No. 3, June 2017, pp. 1489-1503.

[10] R. Liu, Z. Sun and D. Ye, “Adaptive Sliding mode Control for Spacecraft Autonomous Rendezvous with Elliptical Orbits and Thrusters Faults", Special section on recent Advances in Fault Diagnosis and Fault-Tolerant Control of Aerospace Engineering Systems, Vol. 5, 2017, pp. 24853-24862.

[11] Z. Guang, L. Yuyang and B. Xingzi, "Conservative term Constrained Kalman Filter for Autonomous Orbit Determination", IEEE Trans. on Aerospace and Electronic Systems, Vol. 54, No. 2, April 2018, pp. 783-793.

[12] X. Zhong, X. Shao, X. Li, Z. Ma and G. Sun, “Fractional order Adaptive Sliding Mode Control for the Deployment of Space Tethered System with Input limitation", IEEE, Vol. 6, 2018, pp. 48958-48969.

[13] C. Floris, "Mean Square Stability of a Second-order Parametric Linear System excited by a Colored Gaussian noise", Journal of Sound and Vibration, vol. 336, 2015, pp. 82-95.

[14] W. C. Xie and R. M. C. So, "Parametric Resonance of a Two-Dimensional System under Bounded Noise Excitation", Nonlinear Dynamics, vol. 36, 2004, pp. 437-453.

[15] W. M. Zhang, G. Meng and Z. K. Peng, “Nonlinear Dynamic Analysis of Atomic Force Microscopy Under Bounded Noise Parametric Excitation", IEEE/ASME Trans. on Mechatronic, vol. 16, no. 6, December 2011, pp. 1063-1072.

[16] P. Kozic, G. Janevski and R. Pavlovic, "Moment Lyapunov Exponents and Stochastic Stability for two Coupled Oscillators", Journal of Mechanics of Materials and Structures, vol. 4, no. 10, 2009, pp. 1689-1701.

[17] P. E. Kloeden and E. Platen, “Numerical Solution of Stochastic Differential Equations", Springer.

[18] G. Kopasakis, Atmospheric Turbulence Modeling for Aero vehicles : Fractional order fits, NASA Center for Aerospace Information, Dec. 2010, pp. 1-9. 